\title{
HÖLDER ESTIMATES FOR SOLUTIONS OF DEGENERATE NONDIVERGENCE ELLIPTIC AND PARABOLIC EQUATIONS
}

\author{
A. I. NAZAROV
}

To my teacher Nina Nikolaevna Ural'tseva on the occasion of her birthday.

\begin{abstract}
We deal with a class of nondivergence type elliptic and parabolic equations degenerating at the coordinate hyperplanes. Assuming that the degeneration is coordinatewise and varies regularly, we prove the Hölder continuity of solutions. Also, the approximative solutions are considered.
\end{abstract}

\section{§1. INTRODUCTION}

Let $n \geq 2$, and let $\Omega$ be a bounded domain in $\mathbb{R}^{n}$ containing the origin. We consider an elliptic equation of nondivergence form

$$
\mathcal{L} u \equiv-\sum_{i, j=1}^{n} a_{i j}(x) u_{x_{i} x_{j}}=f(x) \text { in } \Omega,
$$

with measurable coefficients. We suppose that a symmetric matrix $\mathcal{A}=\left(a_{i j}\right)$ has diagonal degeneration at coordinate hyperplanes $x_{k}=0, k=1, \ldots, n$. Namely, we assume that

$$
\mathcal{A}=\sqrt{\Lambda} \tilde{\mathcal{A}} \sqrt{\Lambda},
$$

where $\tilde{\mathcal{A}}$ is a measurable matrix function satisfying the uniform ellipticity condition; i.e., for all $x \in \Omega$ we have

$$
\nu|\xi|^{2} \leq(\tilde{\mathcal{A}} \xi, \xi) \leq \nu^{-1}|\xi|^{2}, \quad \xi \in \mathbb{R}^{n}, \quad \nu>0
$$

Next, $\Lambda$ is a diagonal matrix of specific form responsible for degeneration:

$$
\Lambda(x)=\operatorname{diag}\left\{\lambda_{1}\left(\left|x_{1}\right|\right), \lambda_{2}\left(\left|x_{2}\right|\right), \ldots, \lambda_{n}\left(\left|x_{n}\right|\right)\right\} .
$$

We obtain local a priori estimates of the Hölder norm for solutions of (1.1) and for solutions of the corresponding parabolic equation

$$
\left.\mathcal{M} u \equiv \partial_{t} u-\sum_{i, j=1}^{n} a_{i j}(x, t) u_{x_{i} x_{j}}=f(x, t) \quad \text { in } \quad Q=\Omega \times\right] 0, T[.
$$

For uniformly elliptic and uniformly parabolic equations such estimates were established in the classical paper [1]; see also [2]. In [3], this result was generalized to the equations with unbounded lower-order coefficients. Another method for obtaining Hölder estimates was proposed in [4. The Hölder continuity of solutions of some nonuniformly elliptic (parabolic) equations was proved in $[5]$.

2010 Mathematics Subject Classification. Primary 35B45; Secondary 35J70, $35 \mathrm{~K} 65$.

Key words and phrases. Nondivergence equations, Hölder estimates, degeneration, regularly varying functions.

The paper is supported by the grant NSh.227.2008.1 and by RFBR grant 08-01-00748. 
Equations with diagonal degeneration (1.1)-(1.4) have been studied in some particular cases since the 1960s, see, e.g., 6, but only for a smooth coefficient matrix $\widetilde{\mathcal{A}}$. Recently, these equations with only measurable coefficients have become of growing interest. One of the reasons is the active development of the theory of free boundary problems related to Gaussian curvature flows (see [7] and also [8, 9, 10]).

In [11, the Harnack inequality and Hölder estimates were obtained for solutions of an equation that can be reduced to (1.1)-(1.4) with

$$
n=2, \quad \lambda_{1}(t) \equiv 1, \quad \lambda_{2}(t)=t^{\alpha}, \quad \alpha<1 .
$$

It is important that in [11] equation (1.1) was considered in a domain located in the half-space $x_{2}>0$. Moreover, in essence, the Neumann condition was imposed on $\partial \Omega \cap$ $\left\{x_{2}=0\right\}$. Thus, the even reflection with respect to $x_{2}=0$ reduces this problem to that of ours. On the other hand, a barrier function constructed in [11 is not available when a degeneration plane intersects the domain. It should be noted that the validity of the corresponding results of [11] for parabolic equations is doubtful. The point is that there is a gap in the proof of Theorem 3.2 in [11] (a version of the parabolic Aleksandrov-type maximum principle). Namely, it should be $\rho^{\frac{1}{3}}$ in place of $\rho^{\frac{2}{3}}$ on the right-hand side of the $\sup u_{+}$estimate. Since the proofs of subsequent statements in the parabolic case are omitted, it is not clear whether they remain valid after this correction.

In the paper [12] (see also [13]) the Hölder continuity of approximative solutions of the homogeneous equation (1.1)-(1.4) was proved under the condition

$$
\lambda_{k}(t)=t^{\alpha_{k}}, \quad-\frac{1}{n-1}<\alpha_{k}<1, \quad k=1, \ldots, n .
$$

The method of [12, 13] develops the techniques of [4] and is based on the Green function estimates for the uniformly elliptic equations with smooth coefficients approximating the equation conjugate to (1.1). Substantial analytic difficulties occur along this way, and the result was obtained only under hard restrictions on $\alpha_{k}$.

We establish the Hölder continuity of solutions of (1.1) and (1.5) under the condition of a regular behavior of the functions $\lambda_{k}$ at a neighborhood of the origin; see $\S 2$. In particular, the functions $\lambda_{k}(t)=t^{\alpha_{k}}$ are admissible for arbitrary $\alpha_{k}<1$.

Our method is based on the classical barrier techniques. To construct the barriers, we uniformize the equation and reduce it to a uniformly elliptic (uniformly parabolic) equation with lower-order terms having strong singularities at the coordinate hyperplanes. For the reduced equations, barrier functions are constructed. The point is that our estimates for these barriers are uniform with respect to the distance from the coordinate hyperplanes. As we show, this approach can easily be modified to cover approximative solutions.

The paper is organized as follows. In $\S 2$, we collect auxiliary lemmas about regularly varying functions. $\S 3$ is devoted to uniformization of the equation and to barrier functions. In $\S 4$, Hölder continuity is proved for solutions of elliptic equations, and in $\S 5$ this is done for solutions of parabolic equations. Finally, in $\S 6$ we consider approximative solutions.

We introduce some notation. $K_{\rho}(x)$ stands for the $n$-dimensional cube centered at $x$, with $2 \rho$-long edges parallel to coordinate axes; $K_{\rho}=K_{\rho}(0)$. If $x=\left(x_{1}, \bar{x}\right)$ (i.e., $\bar{x}$ is the projection of $x$ to the hyperplane $\left.x_{1}=0\right)$, then $K_{\rho}^{\prime}(\bar{x})$ stands for the $(n-1)$-dimensional projection of $K_{\rho}(x)$.

For a set $E$, we denote by $|E|$ its $n$-dimensional $((n+1)$-dimensional in $\S 5)$ Lebesgue measure. We also set $E^{+}=E \cap \mathbb{R}_{+}^{n}$, where $\mathbb{R}_{+}^{n}$ is the positive orthant in $\mathbb{R}^{n}$.

We put $f_{ \pm}=\max \{ \pm f, 0\}$. If $u$ is a continuous function, then $A_{s}^{u}=\{x: u(x)>s\}$ stands for its level set. 


\section{§2. About Regularly VARYing FunCtions}

Recall that a positive function $\varphi(\tau), \tau>0$, is regularly varying of order $\alpha$ near the origin 1 (we shall write $\varphi \in \mathcal{R}_{\alpha}$ ) if for any $c>0$ we have

$$
\varphi(c \tau) / \varphi(\tau) \rightarrow c^{\alpha} \quad \text { as } \quad \tau \rightarrow+0 .
$$

In a similar way we define regularly varying functions near infinity.

The properties of regularly varying functions are well known. We list some of them that we shall use. The proofs can be found, for example, in the classical book [14].

Proposition 2.1. 1. If $\varphi \in \mathcal{R}_{\alpha}$, then for all $0<c_{1}<c_{2}<+\infty$ the limit in (2.1) is uniform with respect to $c \in\left[c_{1}, c_{2}\right]$.

2. If $\varphi_{1} \in \mathcal{R}_{\alpha_{1}}, \varphi_{2} \in \mathcal{R}_{\alpha_{2}}$, then $\varphi_{1} \varphi_{2} \in \mathcal{R}_{\alpha_{1}+\alpha_{2}}$. If $\varphi \in \mathcal{R}_{\alpha}$, then $1 / \varphi \in \mathcal{R}_{-\alpha}$.

3. If $\varphi_{1} \in \mathcal{R}_{\alpha_{1}}, \varphi_{2} \in \mathcal{R}_{\alpha_{2}}$, and $\alpha_{1}>0$, then $\varphi_{2} \circ \varphi_{1} \in \mathcal{R}_{\alpha_{1} \alpha_{2}}$. In particular, $\varphi_{1}^{\alpha_{2}} \in \mathcal{R}_{\alpha_{1} \alpha_{2}}$.

4. If $\varphi \in \mathcal{R}_{\alpha}, \alpha>0$, then $\varphi$ increases monotonically in some neighborhood of the origin, and $\varphi^{-1} \in \mathcal{R}_{1 / \alpha}$.

5. If $\varphi \in \mathcal{R}_{\alpha}, \alpha>-1$, then the integral $\psi(t)=\int_{0}^{t} \varphi(\tau) d \tau$ converges, and $\psi \in \mathcal{R}_{\alpha+1}$.

6. If $\varphi \in \mathcal{C}^{1}$, and $\tau \varphi^{\prime}(\tau) / \varphi(\tau) \rightarrow \alpha$ as $\tau \rightarrow+0$, then $\varphi \in \mathcal{R}_{\alpha}$. Moreover, if $\varphi \in \mathcal{R}_{\alpha}$, then there exists a continuously differentiable function $\varphi_{1} \in \mathcal{R}_{\alpha}$ such that

$$
\frac{\varphi_{1}(\tau)}{\varphi(\tau)} \rightarrow 1, \quad \frac{\tau \varphi_{1}^{\prime}(\tau)}{\varphi_{1}(\tau)} \rightarrow \alpha \quad \text { as } \quad \tau \rightarrow+0
$$

In what follows we need some statements about measures generated by regularly varying densities. The first statement resembles the well-known Chebyshev inequality.

Lemma 2.2. Suppose $\varphi_{k} \in \mathcal{R}_{\alpha_{k}}, k=1,2$, with $\alpha_{1}>-1, \alpha_{2}>-1, \alpha_{1}+\alpha_{2}>-1$. For $-1 \leq t_{1}<t_{2} \leq 1$ we have

$$
\frac{\left(t_{2}-t_{1}\right) \int_{t_{1}}^{t_{2}} \varphi_{1}(|\tau|) \varphi_{2}(|\tau|) d \tau}{\int_{t_{1}}^{t_{2}} \varphi_{1}(|\tau|) d \tau \cdot \int_{t_{1}}^{t_{2}} \varphi_{2}(|\tau|) d \tau} \asymp 1
$$

Proof. Without loss of generality, $t_{2} \geq\left|t_{1}\right|$. Note that, replacing $\varphi_{1}$ and $\varphi_{2}$ with functions equivalent to them at the origin, we keep relation (2.3). Thus, part 6 of Proposition 2.1 allows us to assume that the functions $\varphi_{1}$ and $\varphi_{2}$ are smooth, and

$$
\lim _{t \rightarrow+0} \frac{t \varphi_{1}^{\prime}(t)}{\varphi_{1}(t)}=\alpha_{1}, \quad \lim _{t \rightarrow+0} \frac{t \varphi_{2}^{\prime}(t)}{\varphi_{2}(t)}=\alpha_{2} .
$$

By Proposition 2.1, parts 2 and 5, the functions

$$
\psi_{1}(t)=\int_{0}^{t} \varphi_{1}(\tau) d \tau, \quad \psi_{2}(t)=\int_{0}^{t} \varphi_{2}(\tau) d \tau, \quad \psi(t)=\int_{0}^{t} \varphi_{1}(\tau) \varphi_{2}(\tau) d \tau
$$

are regularly varying with exponents $\alpha_{1}+1, \alpha_{2}+1$, and $\alpha_{1}+\alpha_{2}+1$, respectively. We take a number $N \geq 3$ such that

$$
N^{\alpha_{1}+1} \geq 4, \quad N^{\alpha_{2}+1} \geq 4, \quad N^{\alpha_{1}+\alpha_{2}+1} \geq 4 .
$$

Upon equivalent replacement, we may assume that

$$
\frac{\psi_{1}(N t)}{\psi_{1}(t)} \geq \frac{N^{\alpha_{1}+1}}{2}, \quad \frac{\psi_{2}(N t)}{\psi_{2}(t)} \geq \frac{N^{\alpha_{2}+1}}{2}, \quad \frac{\psi(N t)}{\psi(t)} \geq \frac{N^{\alpha_{1}+\alpha_{2}+1}}{2}
$$

\footnotetext{
${ }^{1}$ For $\alpha=0$ such a function is also called a slowly varying function near the origin.
} 
for $0<t \leq 1 / N$. Therefore, if $t_{2} \geq N t_{1}$, then

$$
\begin{aligned}
\frac{\left(t_{2}-t_{1}\right) \int_{t_{1}}^{t_{2}} \varphi_{1}(|\tau|) \varphi_{2}(|\tau|) d \tau}{\int_{t_{1}}^{t_{2}} \varphi_{1}(|\tau|) d \tau \cdot \int_{t_{1}}^{t_{2}} \varphi_{2}(|\tau|) d \tau} & \asymp \frac{t_{2} \psi\left(t_{2}\right)}{\psi_{1}\left(t_{2}\right) \psi_{2}\left(t_{2}\right)} \\
& =\frac{\psi\left(t_{2}\right)}{t_{2} \varphi_{1}\left(t_{2}\right) \varphi_{2}\left(t_{2}\right)} \cdot \frac{t_{2} \varphi_{1}\left(t_{2}\right)}{\psi_{1}\left(t_{2}\right)} \cdot \frac{t_{2} \varphi_{2}\left(t_{2}\right)}{\psi_{2}\left(t_{2}\right)} \asymp 1
\end{aligned}
$$

(the first relation is implied by (2.5) and (2.6), and the last one follows from the fact that, by $(2.4)$, the limit of this product at zero equals $\left.\frac{\left(\alpha_{1}+1\right)\left(\alpha_{2}+1\right)}{\alpha_{1}+\alpha_{2}+1}\right)$.

Now, if $t_{2}<N t_{1}$, then, by the Lagrange theorem, for some $1<\theta, \theta_{1}, \theta_{2}<N$ we have

$$
\frac{\left(t_{2}-t_{1}\right) \int_{t_{1}}^{t_{2}} \varphi_{1}(|\tau|) \varphi_{2}(|\tau|) d \tau}{\int_{t_{1}}^{t_{2}} \varphi_{1}(|\tau|) d \tau \cdot \int_{t_{1}}^{t_{2}} \varphi_{2}(|\tau|) d \tau}=\frac{\varphi_{1}\left(\theta t_{1}\right) \varphi_{2}\left(\theta t_{1}\right)}{\varphi_{1}\left(\theta_{1} t_{1}\right) \varphi_{2}\left(\theta_{2} t_{1}\right)} \asymp 1
$$

(the last relation follows from part 1 in Proposition 2.1).

The next lemma claims that if a set is "thin" with respect to Lebesgue measure, then it is also "thin" with respect to a measure with regularly varying density.

Lemma 2.3. Suppose $\varphi_{k} \in \mathcal{R}_{\alpha_{k}}, \alpha_{k}>-1, k=1, \ldots, n$. For measurable sets $E \subset \mathbb{R}^{n}$, we define the measure

$$
|E|_{*}=\int_{E} \varphi_{1}\left(\left|x_{1}\right|\right) \ldots \varphi_{n}\left(\left|x_{n}\right|\right) d x_{1} \ldots d x_{n} .
$$

Then, for any $\varepsilon>0$ there exists $\delta>0$ depending only on $\varepsilon$ and the collection $\left(\varphi_{k}\right)$ such that for all cubes $K_{\rho}\left(x^{0}\right) \subset K_{1}$ and arbitrary measurable set $E$ we have

$$
\left|E \cap K_{\rho}\left(x^{0}\right)\right| \leq \delta \cdot\left|K_{\rho}\left(x^{0}\right)\right| \Longrightarrow\left|E \cap K_{\rho}\left(x^{0}\right)\right|_{*} \leq \varepsilon \cdot\left|K_{\rho}\left(x^{0}\right)\right|_{*} .
$$

Proof. Without loss of generality, we may assume that $x^{0} \in \mathbb{R}_{+}^{n}$. We take a number $N \geq 3$, such that $N^{\alpha_{k}+1} \geq 4, k=1, \ldots, n$. Suppose, also without loss of generality, that the point $x^{0}$ is located "near" the coordinate hyperplanes $x_{k}=0$ with $k \leq m$ and "far" from the coordinate hyperplanes $x_{k}=0$ with $k>m$ (here $0 \leq m \leq n$ ). Namely,

$$
x_{k}^{0} \leq \frac{N+1}{N-1} \cdot \rho, \quad k=1, \ldots, m ; \quad x_{k}^{0}>\frac{N+1}{N-1} \cdot \rho, \quad k=m+1, \ldots, n .
$$

Note that replacing $\varphi_{k}$ with equivalent functions leads to multiplication of the measure $|E|_{*}$ of any set $E$ by a quantity bounded by positive constants from above and from below. So, this does not influence the claim. Therefore, we may assume that the functions $\psi_{k}(t)=\int_{0}^{t} \varphi_{k}(\tau) d \tau$ satisfy

$$
\frac{\psi_{k}(N t)}{\psi_{k}(t)} \geq \frac{N^{\alpha_{k}+1}}{2}, \quad 0<t \leq 1 / N .
$$

Inequalities (2.7) and (2.8) imply that

$$
\int_{x_{k}^{0}-\rho}^{x_{k}^{0}+\rho} \varphi_{k}(\tau) d \tau \asymp \begin{cases}\psi_{k}(\rho) \asymp \rho \varphi_{k}(\rho) & \text { if } k=1, \ldots, m, \\ \rho \varphi_{k}\left(x_{k}^{0}\right) & \text { if } k=m+1, \ldots, n,\end{cases}
$$

whence

$$
\left|K_{\rho}\left(x^{0}\right)\right|_{*} \asymp \rho^{n} \varphi_{1}(\rho) \ldots \varphi_{m}(\rho) \varphi_{m+1}\left(x_{m+1}^{0}\right) \ldots \varphi_{n}\left(x_{n}^{0}\right) .
$$

Now we estimate the measure $\left|E \cap K_{\rho}\left(x^{0}\right)\right|_{*}$. By the "bathtub principle" (see, e.g., [15, Theorem 1.14]), we conclude that if the measure $\left|E \cap K_{\rho}\left(x^{0}\right)\right|$ is fixed, then the maximal value of $\left|E \cap K_{\rho}\left(x^{0}\right)\right|_{*}$ is attained if $E$ is the level set

$$
E_{s}=\left\{x: \varphi_{1}\left(\left|x_{1}\right|\right) \ldots \varphi_{n}\left(\left|x_{n}\right|\right)>s\right\}
$$


with a suitably chosen $s$ (replacing the $\varphi_{k}$ with equivalent functions, we may assume without loss of generality that all sets $\left\{\varphi_{1}\left(\left|x_{1}\right|\right) \ldots \varphi_{n}\left(\left|x_{n}\right|\right)=s\right\}$ have zero measure).

Obviously, $K_{\rho}\left(x^{0}\right)$ lies in the parallelepiped

$$
\mathcal{P}(\rho)=\left\{x:\left|x_{k}\right|<3 \rho, k \leq m ; x_{k}^{0}-\rho<x_{k}<x_{k}^{0}+\rho, k>m\right\} .
$$

On the other hand, from (2.10) we see that $\left|K_{\rho}\left(x^{0}\right)\right|_{*} \asymp|\mathcal{P}(\rho)|_{*}$. Therefore, it suffices to estimate $\left|E_{s} \cap \mathcal{P}(\rho)\right|_{*}$, and even $\left|E_{s} \cap \mathcal{P}^{+}(\rho)\right|_{*}$, by symmetry.

We introduce the sets

$$
\widetilde{E}_{1}(\widehat{\delta}, \rho)=\mathcal{P}^{+}(\rho) \cap\left\{x: x_{k}>\widehat{\delta} \rho, k \leq m\right\} ; \quad \widetilde{E}_{2}(\widehat{\delta}, \rho)=\mathcal{P}^{+}(\rho) \backslash \widetilde{E}_{1}(\widehat{\delta}, \rho) .
$$

Then, obviously, $\left|E_{s} \cap \mathcal{P}^{+}(\rho)\right|_{*} \leq\left|E_{s} \cap \widetilde{E}_{1}(\widehat{\delta}, \rho)\right|_{*}+\left|\widetilde{E}_{2}(\widehat{\delta}, \rho)\right|_{*}$.

Relations (2.9) and (2.10) imply that, for sufficiently small $\hat{\delta}$, we have

$$
\left|\widetilde{E}_{2}(\widehat{\delta}, \rho)\right|_{*} \asymp\left|K_{\rho}\left(x^{0}\right)\right|_{*} \cdot \sum_{k=1}^{m} \frac{\psi_{k}(\widehat{\delta} \rho)}{\psi_{k}(\rho)} .
$$

Therefore, for any $\varepsilon>0$ there exists $\widehat{\delta}>0$, depending only on $\varepsilon$ and $\left(\varphi_{k}\right)$, such that $\left|\widetilde{E}_{2}(\widehat{\delta}, \rho)\right|_{*} \leq \frac{\varepsilon}{2}\left|K_{\rho}\left(x^{0}\right)\right|_{*}$.

Next, in the segment $[\widehat{\delta}, 1]$ we have $\varphi_{k}(\rho z) \asymp z^{\alpha_{k}} \varphi_{k}(\rho)$. Hence, using the second line in (2.9), we obtain the following relation on the set $E_{1}(\widehat{\delta}, \rho)$ (here $z_{k}=\frac{x_{k}}{\rho}, \hat{N}$ depends only on $\widehat{\delta}$ and $\left(\varphi_{k}\right)$, and $\mu$ stands for an arbitrary positive number):

$$
\begin{aligned}
z_{1}^{\alpha_{1}} \ldots & z_{m}^{\alpha_{m}}>\hat{N} \mu \\
& \Longrightarrow \varphi_{1}\left(x_{1}\right) \ldots \varphi_{n}\left(x_{n}\right)>\mu \varphi_{1}(\rho) \ldots \varphi_{m}(\rho) \varphi_{m+1}\left(x_{m+1}^{0}\right) \ldots \varphi_{n}\left(x_{n}^{0}\right) \\
& \Longrightarrow z_{1}^{\alpha_{1}} \ldots z_{m}^{\alpha_{m}}>\frac{\mu}{\widehat{N}} .
\end{aligned}
$$

We set $s=\mu \varphi_{1}(\rho) \ldots \varphi_{m}(\rho) \varphi_{m+1}\left(x_{m+1}^{0}\right) \ldots \varphi_{n}\left(x_{n}^{0}\right)$. Then

$$
\begin{aligned}
& \left|E_{s} \cap \widetilde{E}_{1}(\widehat{\delta}, \rho)\right|_{*} \leq \int_{\substack{z_{1}^{\alpha_{1}} \ldots \\
x_{k} \geq \hat{\delta} \rho, k=1, \ldots, m \\
x \in \mathcal{P}^{+}(\rho)}} \varphi_{1}^{z_{m}>\mu / \widehat{N}}\left(x_{1}\right) \ldots \varphi_{n}\left(x_{n}\right) d x_{1} \ldots d x_{n} \\
& \leq \hat{N} \rho^{n} \varphi_{1}(\rho) \ldots \varphi_{m}(\rho) \varphi_{m+1}\left(x_{m+1}^{0}\right) \ldots \varphi_{n}\left(x_{n}^{0}\right) . \quad \int \quad z_{1}^{\alpha_{1}} \ldots z_{m}^{\alpha_{m}} d z_{1} \ldots d z_{m} . \\
& \begin{array}{c}
z_{1}^{\alpha_{1}} \ldots z_{m}^{\alpha_{m}}>\mu / \widehat{N} \\
z_{k} \leq 1, k=1, \ldots, m
\end{array}
\end{aligned}
$$

By (2.10), there exists $\mu$, depending only on $\varepsilon, \hat{N}$, and $\alpha_{k}, k=1, \ldots, m$, such that $\left|E_{s} \cap \widetilde{E}_{1}(\widehat{\delta}, \rho)\right|_{*} \leq \frac{\varepsilon}{2}\left|K_{\rho}\left(x^{0}\right)\right|_{*}$. We take the smallest admissible $\mu$ and take $\delta$ depending on $\mu, \hat{N}$, and the collection $\left(\alpha_{k}\right)$ such that $\left|K_{1}^{+} \cap\left\{z: z_{1}^{\alpha_{1}} \ldots z_{m}^{\alpha_{m}}>\mu / \hat{N}\right\}\right| \geq \delta$ for any $m \leq n$ and for any choice of $m$ exponents $\alpha_{k}$ out of $n$. This completes the proof.

\section{§3. COORDinate transformation AND BARriers}

We suppose that the functions in (1.4) satisfy

$$
\lambda_{k} \in \mathcal{R}_{\alpha_{k}}, \quad \alpha_{k}<1, \quad k=1, \ldots, n .
$$

Note that, after replacing $\lambda_{k}$ with functions equivalent at zero, equation (1.1) keeps its structure, and the constant $\nu$ in (1.3) can be controlled. Thus, by part 6 of Proposition 2.1, we may assume without loss of generality that $\left.\left.\lambda_{k} \in \mathcal{C}^{1}(] 0,1\right]\right)$ and $t \lambda_{k}^{\prime}(t) / \lambda_{k}(t) \rightarrow$ $\alpha_{k}$ as $t \rightarrow+0$. We extend the $\lambda_{k}$ to the negative half-axis as even functions. 
Next, since Hölder continuity is a local property, it suffices to establish this property in a cube $K_{R_{0}}\left(x^{0}\right) \Subset \Omega$ with a small (given) edge of length $2 R_{0}$ and arbitrary center $x^{0}$. At the same time, if $x^{0}$ is situated "far" from the $k$ th coordinate hyperplane (i.e., $\left|x_{k}^{0}\right| \geq 2 R_{0}$ ), we may assume (shifting the origin and changing $\nu$ if needed) that $\lambda_{k} \equiv 1$ and $x_{k}^{0}=0$. Thus, it suffices to consider the case where $x^{0}$ is "near" the origin (i.e., $\left.\left|x_{k}^{0}\right|<2 R_{0}, k=1, \ldots, n\right)$.

Remark 1. Some regularly varying functions arise in this section by transformation of $\lambda_{k}$. Also we consider some terms with finite limits at zero. It is easily seen that there exists $R_{0} \leq 1$, depending only on $\left(\lambda_{i}\right)$, such that if $\left|x_{k}\right|<3 R_{0}$, then all such terms are "closely approximated" by their limit values (surely, this statement needs to be specified in each specific case).

We introduce a new coordinate system:

$$
y_{k}=\int_{0}^{x_{k}} \frac{d \tau}{\sqrt{\lambda_{k}(\tau)}}, \quad k=1, \ldots, n .
$$

Obviously, the transformation (3.2) (as well as its inverse) satisfies the Hölder condition in $\bar{\Omega}$. Thus, the Hölder exponent and Hölder constant of an arbitrary function in the original coordinates depend only on its Hölder exponent (respectively, constant) in the new coordinates and on the collection $\left(\lambda_{i}\right)$.

In the $y$-coordinates, equation (1.1) is written as follows (we keep the original notation for the "transplanted" functions):

$$
\widetilde{\mathcal{L}} u \equiv-\sum_{i, j=1}^{n} \tilde{a}_{i j}(y) u_{y_{i} y_{j}}+\sum_{i=1}^{n} \tilde{a}_{i i}(y) \beta_{i}\left(y_{i}\right) u_{y_{i}}=f(y),
$$

where

$$
\beta_{k}\left(y_{k}\right)=\frac{\lambda_{k}^{\prime}\left(x_{k}\right)}{2 \sqrt{\lambda_{k}\left(x_{k}\right)}}
$$

Note that

$$
\left.\sigma_{k} \equiv \lim _{y_{k} \rightarrow 0} y_{k} \beta_{k}\left(y_{k}\right)=\lim _{x_{k} \rightarrow 0} \frac{x_{k} \lambda^{\prime}\left(x_{k}\right)}{\lambda_{k}\left(x_{k}\right)} \cdot \lim _{x_{k} \rightarrow 0} \frac{\int_{0}^{x_{k}} \frac{d \tau}{\sqrt{\lambda_{k}(\tau)}}}{2 x_{k} / \sqrt{\lambda_{k}\left(x_{k}\right)}}=\frac{\alpha_{k}}{2-\alpha_{k}} \in\right]-1,1[.
$$

By Remark 1, we may assume that if $\left|x_{k}\right|<3 R_{0}$, then

$$
\sigma_{k}-\frac{1-\left|\sigma_{k}\right|}{2} \leq y_{k} \beta_{k}\left(y_{k}\right) \leq \sigma_{k}+\frac{1-\left|\sigma_{k}\right|}{2} .
$$

The image of the cube $K_{3 R_{0}}$ under the transformation (3.2) is a rectangular parallelepiped to be denoted by $\widetilde{\Pi}$.

Now we introduce a set of "one-dimensional" functions that will be the base for constructing barriers.

Lemma 3.1. Let $K_{\rho}\left(y^{0}\right) \subset \widetilde{\Pi}$. Then there exist $B_{k}$, depending only on $y_{k}^{0}$ and $\lambda_{k}$ $(k=1, \ldots, n)$, such that the functions $w_{k}\left(y_{k}\right)=-y_{k}^{2}+B_{k} x_{k}$ (here $x_{k}\left(y_{k}\right)$ stands for the function inverse to (3.2)) satisfy the following relations:

1) $0<\nu\left(1-\left(\sigma_{k}\right)_{+}\right) \leq \widetilde{\mathcal{L}} w_{k} \leq 4 \nu^{-1}$ in $K_{\rho}\left(y^{0}\right)$;

2) $w_{k}$ attains its maximum at $y_{k}^{0}$; moreover,

$$
C_{1} \rho^{2} \leq w_{k}\left(y_{k}^{0}\right)-w_{k}\left(y_{k}^{0}+\rho\right) \leq C_{2} \rho^{2} ; \quad C_{1} \rho^{2} \leq w_{k}\left(y_{k}^{0}\right)-w_{k}\left(y_{k}^{0}-\rho\right) \leq C_{2} \rho^{2},
$$

where $C_{1}, C_{2}$ are positive constants depending only on the collection $\left(\lambda_{i}\right)$; 
3) there exists $\vartheta>0$, depending only on $\left(\lambda_{i}\right)$, such that if $\left|1-\frac{\rho}{y_{k}^{0}}\right| \leq \vartheta$, then

$$
\widehat{C}_{1} \rho^{2} \leq w_{k}\left(y_{k}^{0}\right)-w_{k}\left(y_{k}^{0}-\rho\right) \leq \widehat{C}_{2} \rho^{2},
$$

where $\widehat{C}_{1}, \widehat{C}_{2}$ are constants depending only on $\left(\lambda_{i}\right)$, and $\widehat{C}_{2} \leq \frac{5}{4} \widehat{C}_{1}$.

Proof. A direct calculation gives $\widetilde{\mathcal{L}}\left(-y_{k}^{2}\right)=2 \widetilde{a}_{k k}\left(1-y_{k} \beta_{k}\left(y_{k}\right)\right)$. Since $\mathcal{L} x_{k}=0$, we see that, by (3.4), property 1 is satisfied for any choice of $B_{k}$.

Next, we note that for $y_{k}^{0}=0$ we can put $B_{k}=0$, and property 2 becomes trivial. Otherwise, if $y_{k}^{0} \neq 0$, then by symmetry we may assume that $y_{k}^{0}>0$. Since $d x_{k} / d y_{k}=$ $\sqrt{\lambda_{k}\left(x_{k}\right)}$, the condition $w_{k}^{\prime}\left(y_{k}^{0}\right)=0$ will be satisfied if we put $B_{k}=\frac{2 y_{k}^{0}}{\sqrt{\lambda_{k}\left(x_{k}\left(y_{k}^{0}\right)\right)}}$. It should be noted that, by parts $3-5$ of Proposition 2.1,

$$
\frac{y_{k}}{\sqrt{\lambda_{k}\left(x_{k}\left(y_{k}\right)\right)}} \in \mathcal{R}_{1-\sigma_{k}} \text { is a monotone increasing function, }
$$

i.e., $B_{k}$ depends on $y_{k}^{0}$ monotonically; thus, for $B_{k}$ fixed, the function $w_{k}$ increases on ]$-\infty, y_{k}^{0}[$ and decreases on $] y_{k}^{0},+\infty[$.

We have

$$
w_{k}\left(y_{k}^{0}\right)-w_{k}\left(y_{k}^{0}+\rho\right)=\rho^{2}+2 y_{k}^{0}\left(\rho+\frac{x_{k}\left(y_{k}^{0}\right)}{\sqrt{\lambda_{k}\left(x_{k}\left(y_{k}^{0}\right)\right)}}-\frac{x_{k}\left(y_{k}^{0}+\rho\right)}{\sqrt{\lambda_{k}\left(x_{k}\left(y_{k}^{0}\right)\right)}}\right) .
$$

Expanding $x_{k}\left(y_{k}^{0}+\rho\right)$ by the Taylor formula, we get

$$
w_{k}\left(y_{k}^{0}\right)-w_{k}\left(y_{k}^{0}+\rho\right)=\rho^{2}\left(1-\frac{y_{k}^{0}}{2 \sqrt{\lambda_{k}\left(x_{k}\left(y_{k}^{0}\right)\right)}} \cdot \lambda_{k}^{\prime}\left(x_{k}\left(y_{k}^{0}+\theta \rho\right)\right)\right),
$$

where $\theta \in] 0,1[$.

Formula (3.7) yields

$$
\left|\frac{y_{k}^{0} \cdot \lambda_{k}^{\prime}\left(x_{k}\left(y_{k}^{0}+\theta \rho\right)\right)}{2 \sqrt{\lambda_{k}\left(x_{k}\left(y_{k}^{0}\right)\right)}}\right| \leq\left|\left(y_{k}^{0}+\theta \rho\right) \cdot \frac{\lambda_{k}^{\prime}\left(x_{k}\left(y_{k}^{0}+\theta \rho\right)\right)}{2 \sqrt{\lambda_{k}\left(x_{k}\left(y_{k}^{0}+\theta \rho\right)\right)}}\right|=\left|\left(y_{k}^{0}+\theta \rho\right) \beta_{k}\left(y_{k}^{0}+\theta \rho\right)\right|,
$$

whence $\frac{1-\sigma_{k}}{2} \rho^{2} \leq w_{k}\left(y_{k}^{0}\right)-w_{k}\left(y_{k}^{0}+\rho\right) \leq \frac{3-\sigma_{k}}{2} \rho^{2}$.

The second inequality in (3.5) is somewhat more complicated. As in (3.8), we have

$$
w_{k}\left(y_{k}^{0}\right)-w_{k}\left(y_{k}^{0}-\rho\right)=\rho^{2}\left(1-\frac{y_{k}^{0}}{2 \sqrt{\lambda_{k}\left(x_{k}\left(y_{k}^{0}\right)\right)}} \cdot \lambda_{k}^{\prime}\left(x_{k}\left(y_{k}^{0}-\theta \rho\right)\right)\right) .
$$

First, suppose that $\rho \leq \delta y_{k}^{0}$, where $\left.\delta \in\right] 0,1[$ is a quantity to be chosen later. By part 1 of Proposition 2.1, Remark 1, and relation (3.7), we may assume that

$$
\frac{y_{k}^{0} \sqrt{\lambda_{k}\left(x_{k}\left(y_{k}^{0}-\theta \rho\right)\right)}}{\left(y_{k}^{0}-\theta \rho\right) \sqrt{\lambda_{k}\left(x_{k}\left(y_{k}^{0}\right)\right)}} \leq\left(\frac{y_{k}^{0}}{y_{k}^{0}-\theta \rho}\right)^{1-\sigma_{k}} \cdot \frac{4-\left|\sigma_{k}\right|}{3} \leq \frac{4-\left|\sigma_{k}\right|}{3(1-\delta)^{1-\sigma_{k}}}
$$

and therefore

$$
\left|\frac{y_{k}^{0}}{2 \sqrt{\lambda_{k}\left(x_{k}\left(y_{k}^{0}\right)\right)}} \cdot \lambda_{k}^{\prime}\left(x_{k}\left(y_{k}^{0}-\theta \rho\right)\right)\right| \leq \frac{4-\left|\sigma_{k}\right|}{3(1-\delta)^{1-\sigma_{k}}} \cdot\left|\left(y_{k}^{0}-\theta \rho\right) \beta_{k}\left(y_{k}^{0}-\theta \rho\right)\right| .
$$

Choosing $\delta=1-\left(1-\left(1-\left|\sigma_{k}\right|\right)^{2} / 9\right)^{\frac{1}{1-\sigma_{k}}}$, we arrive at

$$
\frac{1-\left|\sigma_{k}\right|}{2+\left|\sigma_{k}\right|} \frac{\rho^{2}}{2} \leq w_{k}\left(y_{k}^{0}\right)-w_{k}\left(y_{k}^{0}-\rho\right) \leq \frac{7+5\left|\sigma_{k}\right|}{2+\left|\sigma_{k}\right|} \frac{\rho^{2}}{2} \text {. }
$$

Now, let $\delta y_{k}^{0} \leq \rho \leq 2 y_{k}^{0}$. Then the above estimate yields

$$
w_{k}\left(y_{k}^{0}\right)-w_{k}\left(y_{k}^{0}-\rho\right) \geq w_{k}\left(y_{k}^{0}\right)-w_{k}\left(y_{k}^{0}-\delta y_{k}^{0}\right) \geq \frac{1-\left|\sigma_{k}\right|}{2+\left|\sigma_{k}\right|} \cdot \frac{\left(\delta y_{k}^{0}\right)^{2}}{2} \geq \frac{1-\left|\sigma_{k}\right|}{2+\left|\sigma_{k}\right|} \cdot \frac{\delta^{2}}{8} \rho^{2} .
$$


On the other hand, $\frac{x_{k}}{\sqrt{\lambda_{k}\left(x_{k}\right)}}=x_{k} \cdot d y_{k} / d x_{k}$, and $y_{k}\left(x_{k}\right) \in \mathcal{R}_{\frac{2-\alpha_{k}}{2}}$. By part 6 of Proposition 2.1 and Remark 1 , we may assume that $B_{k} x_{k}\left(y_{k}^{0}\right) \leq 2\left(2-\alpha_{k}\right)\left(y_{k}^{0}\right)^{2}$, whence $w_{k}\left(y_{k}^{0}\right)-w_{k}\left(y_{k}^{0}-\rho\right) \leq w_{k}\left(y_{k}^{0}\right)-w_{k}\left(-y_{k}^{0}\right)=2 B_{k} x_{k}\left(y_{k}^{0}\right) \leq 4\left(2-\alpha_{k}\right)\left(y_{k}^{0}\right)^{2} \leq 4 \frac{2-\alpha_{k}}{\delta^{2}} \rho^{2}$.

Finally, let $\rho \geq 2 y_{k}^{0}$. Then $-\rho \leq y_{k}^{0}-\rho \leq-\frac{\rho}{2}$, so that

$$
\begin{aligned}
\frac{\rho^{2}}{4} & \leq-w_{k}\left(-\frac{\rho}{2}\right) \leq w_{k}\left(y_{k}^{0}\right)-w_{k}\left(y_{k}^{0}-\rho\right) \\
& \leq w_{k}\left(\frac{\rho}{2}\right)-w_{k}(-\rho) \leq 2 B_{k} x_{k}(\rho)+\rho^{2} \leq\left(9-4 \alpha_{k}\right) \rho^{2} .
\end{aligned}
$$

This completes the proof of part 2 .

Under the conditions of part 3, we have $y_{k}^{0}>0$ and $\left|y_{k}^{0}-\rho\right| \leq \vartheta y_{k}^{0}$. Therefore,

$$
\begin{aligned}
B_{k}\left(x_{k}\left(y_{k}^{0}\right)\right. & \left.-x_{k}\left(\vartheta y_{k}^{0}\right)\right)-\left(1-\vartheta^{2}\right)\left(y_{k}^{0}\right)^{2} \\
& =w_{k}\left(y_{k}^{0}\right)-w_{k}\left(\vartheta y_{k}^{0}\right) \leq w_{k}\left(y_{k}^{0}\right)-w_{k}\left(y_{k}^{0}-\rho\right) \leq w_{k}\left(y_{k}^{0}\right)-w_{k}\left(-\vartheta y_{k}^{0}\right) \\
& =B_{k}\left(x_{k}\left(y_{k}^{0}\right)+x_{k}\left(\vartheta y_{k}^{0}\right)\right)-\left(1-\vartheta^{2}\right)\left(y_{k}^{0}\right)^{2} .
\end{aligned}
$$

Since $x_{k}\left(y_{k}\right) \in \mathcal{R}_{1+\sigma_{k}}$, Remark 1 allows us to assume that $x_{k}\left(\vartheta y_{k}^{0}\right) \leq 2 \vartheta^{1+\sigma_{k}} x_{k}\left(y_{k}^{0}\right)$. Next, by part 6 of Proposition 2.1 and Remark 1, we may assume that

$$
\begin{aligned}
\frac{(1-\vartheta)\left(2-\alpha_{k}\right)}{(1+\vartheta)^{2}} \rho^{2} & \leq(1-\vartheta)\left(2-\alpha_{k}\right)\left(y_{k}^{0}\right)^{2} \leq B_{k} x_{k}\left(y_{k}^{0}\right) \\
& \leq(1+\vartheta)\left(2-\alpha_{k}\right)\left(y_{k}^{0}\right)^{2} \leq \frac{(1+\vartheta)\left(2-\alpha_{k}\right)}{(1-\vartheta)^{2}} \rho^{2} .
\end{aligned}
$$

Consequently,

$$
\left(1-\alpha_{k}-c\left(\vartheta, \sigma_{k}\right)\right) \rho^{2} \leq w_{k}\left(y_{k}^{0}\right)-w_{k}\left(y_{k}^{0}-\rho\right) \leq\left(1-\alpha_{k}+c\left(\vartheta, \sigma_{k}\right)\right) \rho^{2},
$$

where $c \rightarrow 0$ as $\vartheta \rightarrow 0$.

It remains to choose $\vartheta$ so that $c\left(\vartheta, \sigma_{k}\right) \leq \frac{1-\alpha_{k}}{9}$ for all $k=1, \ldots, n$.

\section{$\S 4$. ElLiptic CASE}

In all lemmas of this section we assume that $v \in W_{n}^{2}\left(K_{3 R_{0}}\right)$ is a function that, after passage to the $y$-coordinates, is nonnegative in the cube $K_{\rho}\left(y^{0}\right) \subset \widetilde{\Pi}$ and satisfies the inequality $\widetilde{\mathcal{L}} v \geq-f(y)$ a.e. We introduce the notation $\lambda=\operatorname{det}(\Lambda)=\prod_{k=1}^{n} \lambda_{k}$.

We follow the classical pattern of [3]. The first lemma shows that if in some cube the level set $A_{s}^{v}$ has sufficiently large measure, then some smaller cube lies in the level set $A_{s / 2}^{v}$ (up to correction for the right-hand side of the equation). A folklore title "the thin set lemma" is explained by the fact that the set under the level $s$ is "thin" (i.e., has small measure).

Lemma 4.1. There exist constants $\zeta \in] 0,1[, \eta \in] 0,1\left[, \gamma>0\right.$, and $C_{3}>0$, depending only on $n, \nu$, and the collection $\left(\lambda_{i}\right)$, such that if the inequality $\left|K_{\rho}\left(y^{0}\right) \cap A_{s}^{v}\right| \geq$ $(1-\zeta)\left|K_{\rho}\left(y^{0}\right)\right|$ is fulfilled for some $s>0$, then

$$
v \geq \frac{s}{2}-C_{3} \rho^{\gamma}\left\|\frac{f_{+}}{\sqrt{\lambda}}\right\|_{n, K_{\rho}\left(y^{0}\right)} \quad \text { in } \quad K_{\eta \rho}\left(y^{0}\right) .
$$

Proof. We construct the barrier function

$$
W(y)=\frac{1}{C_{1} \rho^{2}} \sum_{k=1}^{n} w_{k}\left(y_{k}\right)+\widetilde{C}
$$


where the $w_{k}$ are the functions on the cube $K_{\rho}\left(y^{0}\right)$ that were defined in Lemma 3.1, $C_{1}$ is the constant occurring in (3.5), and $\widetilde{C}$ is determined by the condition $W\left(y^{0}\right)=1$. From (3.5) it follows that $\left.W\right|_{\partial K_{\rho}\left(y^{0}\right)} \leq 0$.

We pass to $x$-coordinates. Denote by $x^{0}$ the image of $y^{0}$, and by $\Pi_{\rho}\left(x^{0}\right)$ the image of $K_{\rho}\left(y^{0}\right)$ (this is a cuboid). We keep the original notation for "transplanted" functions.

Obviously, the function $s W(x)-v(x)$ is nonpositive on $\partial \Pi_{\rho}\left(x^{0}\right)$. Applying the Aleksandrov maximum principle (see [16]), we obtain

$$
\begin{aligned}
\left(\max _{\Pi_{\rho}\left(x^{0}\right)}(s W-v)_{+}\right)^{n} & \leq N_{1}(n)\left|\Pi_{\rho}\left(x^{0}\right)\right| \cdot \int_{A_{0}^{s W-v} \cap \Pi_{\rho}\left(x^{0}\right)} \frac{(\mathcal{L}(s W(x)-v(x)))_{+}^{n}}{\operatorname{det}(\mathcal{A})} d x \\
& \leq \frac{N_{1}(n)}{\nu^{n}}\left|\Pi_{\rho}\left(x^{0}\right)\right| \cdot \int_{A_{0}^{s W-v} \cap K_{\rho}\left(y^{0}\right)} \frac{(\widetilde{\mathcal{L}}(s W(y)-v(y)))_{+}^{n}}{\sqrt{\lambda(x(y))}} d y .
\end{aligned}
$$

By part 1 of Lemma 3.1, $\widetilde{\mathcal{L}}(s W-v) \leq \frac{4 n \nu^{-1}}{C_{1} \rho^{2}} s+f_{+}(x)$. Therefore,

$$
(s W-v)_{+} \leq \frac{N_{2}(n)}{\nu}\left|\Pi_{\rho}\left(x^{0}\right)\right|^{\frac{1}{n}} \cdot\left(s \frac{\left|A_{0}^{s W-v} \cap K_{\rho}\left(y^{0}\right)\right|_{*}^{\frac{1}{n}}}{\nu C_{1} \rho^{2}}+\left\|\frac{f_{+}}{\sqrt{\lambda}}\right\|_{n, K_{\rho}\left(y^{0}\right)}\right),
$$

where, as in Lemma 2.3,

$$
|E|_{*}=\int_{E} \frac{d y_{1} \ldots d y_{n}}{\prod_{k=1}^{n} \sqrt{\lambda_{k}\left(x_{k}\left(y_{k}\right)\right)}} .
$$

Since $W \leq 1$, it is easily seen that $\left(A_{0}^{s W-v} \cap K_{\rho}\left(y^{0}\right)\right) \subset K_{\rho}\left(y^{0}\right) \backslash A_{s}^{v}$. Denoting $\varepsilon=\frac{\left|K_{\rho}\left(y^{0}\right) \backslash A_{s}^{v}\right|_{*}}{\left|K_{\rho}\left(y^{0}\right)\right|_{*}}$, we obtain

$$
v(y) \geq s W(y)-\frac{N_{2}(n)}{\nu}\left|\Pi_{\rho}\left(x^{0}\right)\right|^{\frac{1}{n}} \cdot\left(s \frac{\left(\varepsilon\left|K_{\rho}\left(y^{0}\right)\right|_{*}\right)^{\frac{1}{n}}}{\nu C_{1} \rho^{2}}+\left\|\frac{f_{+}}{\sqrt{\lambda}}\right\|_{n, K_{\rho}\left(y^{0}\right)}\right) .
$$

Note that

$$
\frac{\left|\Pi_{\rho}\left(x^{0}\right)\right| \cdot\left|K_{\rho}\left(y^{0}\right)\right|_{*}}{\rho^{2 n}}=\prod_{k=1}^{n} \frac{\int_{y_{k}^{0}-\rho}^{y_{k}^{0}+\rho} \sqrt{\lambda_{k}\left(x_{k}\left(y_{k}\right)\right)} d y_{k} \cdot \int_{y_{k}^{0}-\rho}^{y_{k}^{0}+\rho} \frac{d y_{k}}{\sqrt{\lambda_{k}\left(x_{k}\left(y_{k}\right)\right)}}}{\rho^{2}} .
$$

By Lemma 2.2, the last expression is bounded from above by a constant depending only on the collection $\left(\lambda_{i}\right)$. Therefore, inequalities (4.3) and (3.5) show that, for $y \in K_{\eta \rho}\left(y^{0}\right)$,

$$
v(y) \geq s\left(1-\frac{n C_{2}}{C_{1}} \eta^{2}-N_{3} \varepsilon^{\frac{1}{n}}\right)-N_{4}\left|\Pi_{\rho}\left(x^{0}\right)\right|^{\frac{1}{n}}\left\|\frac{f_{+}}{\sqrt{\lambda}}\right\|_{n, K_{\rho}\left(y^{0}\right)}
$$

(here $C_{2}$ is the constant from (3.5), while $N_{3}$ and $N_{4}$ depend only on $n, \nu$, and $\left(\lambda_{i}\right)$ ).

Since $\sqrt{\lambda_{k}\left(x_{k}\left(y_{k}\right)\right)} \in \mathcal{R}_{\sigma_{k}}$, formula (2.10) yields

$$
\left|\Pi_{\rho}\left(x^{0}\right)\right| \asymp \rho^{n} \prod_{k=1}^{m} \sqrt{\lambda_{k}\left(x_{k}(\rho)\right)} \cdot \prod_{k=m+1}^{n} \sqrt{\lambda_{k}\left(x_{k}\left(y_{k}^{0}\right)\right)} \leq N_{5} \rho^{n \gamma},
$$

where $\gamma=\frac{1}{2}-\frac{1}{2 n} \sum_{k=1}^{n}\left(\sigma_{k}\right)_{-}>0$, and $N_{5}$ depends only on the collection $\left(\lambda_{i}\right)$.

We substitute (4.5) in (4.4) and put $\eta=\sqrt{C_{1} /\left(4 n C_{2}\right)}, C_{3}=N_{4} N_{5}^{\frac{1}{n}}$. It remains to note that, by Lemma 2.3 , the estimate $\left|K_{\rho}\left(y^{0}\right) \backslash A_{s}^{v}\right| \leq \zeta \cdot\left|K_{\rho}\left(y^{0}\right)\right|$ for sufficiently small $\zeta$ ensures that $\varepsilon \leq\left(4 N_{3}\right)^{-n}$, and we arrive at (4.1).

The next lemma shows that a positive lower bound for $v$ on a face of a sufficiently large subcube situated close to a coordinate hyperplane can be transferred across this hyperplane by a thin "bridge". 
Lemma 4.2. There exist constants $\hat{\vartheta} \in] 0, \frac{1}{4}\left[\right.$ and $C_{4}>0$, depending only on $n, \nu$, and $\left(\lambda_{i}\right)$, such that if $0 \leq y_{m}^{0} \leq(1-\widehat{\vartheta}) \rho$ for some $m$ (for definiteness, let $\left.m=1\right)$, and if for some $s>0$ the inequality $v \geq s$ is fulfilled on the set $\left\{\widehat{\vartheta}^{2} \rho\right\} \times K_{(1-\hat{\vartheta}) \rho}^{\prime}\left(\overline{y^{0}}\right)$, then

$$
\left.v \geq \frac{s}{2}-C_{4} \rho^{\gamma}\left\|\frac{f_{+}}{\sqrt{\lambda}}\right\|_{n, K_{\rho}\left(y^{0}\right)} \quad \text { in the cuboid }\right]-\widehat{\vartheta}^{2} \rho, \widehat{\vartheta}^{2} \rho\left[\times K_{\hat{\vartheta}_{\rho}}^{\prime}\left(\overline{y^{0}}\right),\right.
$$

where $\gamma$ is the same constant as in Lemma 4.1.

Proof. Without loss of generality, we may assume that $\widehat{\vartheta} \leq \vartheta$, where $\vartheta$ is the constant occurring in part 3 of Lemma 3.1. We denote $\widehat{y^{0}}=\left(-\widehat{\vartheta} \rho, \overline{y^{0}}\right)$ and define the barrier function

$$
\widehat{W}(y)=\frac{1}{C_{1}((1-\hat{\vartheta}) \rho)^{2}} \sum_{k=2}^{n} w_{k}\left(y_{k}\right)-\frac{w_{1}\left(y_{1}\right)}{\widehat{C}_{1}(\widehat{\vartheta}(\widehat{\vartheta}+1) \rho)^{2}}+\widetilde{C},
$$

where the $w_{k}$ are functions on the cube $K_{\rho}\left(\widehat{y^{0}}\right)$ that were defined in Lemma 3.1, $C_{1}$ and $\widehat{C}_{1}$ are constants from (3.5) and (3.6), respectively, and $\widetilde{C}$ is determined by the condition $\widehat{W}\left(\widehat{\vartheta}^{2} \rho, \overline{y^{0}}\right)=1$.

Consider the following $\widehat{\vartheta}(\widehat{\vartheta}+1) \rho$-thick rectangular parallelepiped adjacent to the hyperplane $y_{1}=\widehat{\vartheta}^{2} \rho$ :

$$
\left.\widetilde{\widehat{\Pi}}_{\rho}=\right]-\hat{\vartheta} \rho, \widehat{\vartheta}^{2} \rho\left[\times K_{(1-\hat{\vartheta}) \rho}^{\prime}\left(\overline{y^{0}}\right) \subset\left(K_{\rho}\left(\widehat{y^{0}}\right) \cap K_{\rho}\left(y^{0}\right)\right) .\right.
$$

Relations (3.5) and (3.6) imply that $\left.\widehat{W}\right|_{\widetilde{\Pi}_{\rho}} \leq 1$, and $\widehat{W} \leq 0$ on $\partial \widetilde{\widehat{\Pi}}_{\rho}$, except for the face $y_{1}=\widehat{\vartheta}^{2} \rho$. Next, by part 1 of Lemma 3.1 ,

$$
\widetilde{\mathcal{L}} \widehat{W} \leq \frac{1}{\rho^{2}} \cdot\left(\frac{4(n-1) \nu^{-1}}{C_{1}(1-\widehat{\vartheta})^{2}}-\frac{\nu\left(1-\left(\sigma_{1}\right)_{+}\right)}{\widehat{C}_{1} \widehat{\vartheta}^{2}(\widehat{\vartheta}+1)^{2}}\right) .
$$

Let $\vartheta_{1}$ denote the positive root of the expression in brackets. Then for $\hat{\vartheta} \leq \vartheta_{1}$ we have $\widetilde{\mathcal{L}} \widehat{W} \leq 0$.

We pass to $x$-coordinates and denote by $\widehat{\Pi}_{\rho}$ the image of $\widetilde{\Pi}_{\rho}$ (it is a cuboid). We keep the original notation for "transplanted" functions.

Obviously, the function $s \widehat{W}(x)-v(x)$ is nonpositive on $\partial \widehat{\Pi}_{\rho}$. Applying the Aleksandrov maximum principle, we obtain

$$
\begin{aligned}
\left(\max _{\widehat{\Pi}_{\rho}}(s W-v)_{+}\right)^{n} & \leq N_{1}(n)\left|\hat{\Pi}_{\rho}\right| \cdot \int_{\hat{\Pi}_{\rho}} \frac{(\mathcal{L}(s W(x)-v(x)))_{+}^{n}}{\operatorname{det}(\mathcal{A})} d x \\
& \leq \frac{N_{1}(n)}{\nu^{n}}\left|\hat{\Pi}_{\rho}\right| \cdot\left\|\frac{f_{+}}{\sqrt{\lambda}}\right\|_{n, \tilde{\Pi}_{\rho}}^{n} .
\end{aligned}
$$

As in (4.5), we have $\left|\hat{\Pi}_{\rho}\right| \leq N_{6} \rho^{n \gamma}$, where $N_{6}$ depends only on the collection $\left(\lambda_{i}\right)$. Recalling (3.5) and (3.6), for $y \in]-\widehat{\vartheta}^{2} \rho, \widehat{\vartheta}^{2} \rho\left[\times K_{\widehat{\vartheta} \rho}^{\prime}\left(\overline{y^{0}}\right)\right.$ we obtain

$$
v(y) \geq s\left[\widehat{W}\left(\widehat{y^{0}}\right)-\frac{(n-1) C_{2} \widehat{\vartheta}^{2}}{C_{1}(1-\widehat{\vartheta})^{2}}+\frac{(1-\widehat{\vartheta})^{2}}{(1+\widehat{\vartheta})^{2}}\right]-C_{4} \rho^{\gamma}\left\|\frac{f_{+}}{\sqrt{\lambda}}\right\|_{n, K_{\rho}\left(y^{0}\right)}
$$

(here $C_{2}$ is the constant from (3.5)).

By (3.6), $\widehat{W}\left(\hat{y}^{0}\right) \geq 1-\frac{\hat{C}_{2}}{\widehat{C}_{1}} \geq-\frac{1}{4}$. Therefore, there exists $\hat{\vartheta} \leq \min \left\{\vartheta, \vartheta_{1}\right\}$ such that the expression in square brackets is at least $\frac{1}{2}$. This gives (4.6). 
The next lemma shows that the positive lower bound for $v$, which was obtained in Lemma 4.1 for a small subcube, can be extended to the entire cube.

Lemma 4.3. There exist constants $\varkappa \in] 0,1\left[\right.$ and $C_{5}>0$, depending only on $n$, $\nu$, and $\left(\lambda_{i}\right)$, such that if the inequality $\left|K_{\frac{\rho}{2}}\left(y^{0}\right) \cap A_{s}^{v}\right| \geq(1-\zeta)\left|K_{\frac{\rho}{2}}\left(y^{0}\right)\right|$ is fulfilled for some $s>0$, then

$$
v \geq \varkappa s-C_{5} \rho^{\gamma}\left\|\frac{f_{+}}{\sqrt{\lambda}}\right\|_{n, K_{\rho}\left(y^{0}\right)} \quad \text { in } \quad K_{\frac{\rho}{2}}\left(y^{0}\right)
$$

(here $\zeta \in] 0,1[$ and $\gamma>0$ are constants from Lemma 4.1).

Proof. Without loss of generality, we may assume that $y^{0} \in \overline{\mathbb{R}_{+}^{n}}$. First, let $f \equiv 0$. Applying Lemma 4.1 to the cube $K_{\frac{\rho}{2}}\left(y^{0}\right)$, we see that $v \geq \frac{s}{2}$ on $K_{\frac{\eta \rho}{2}}\left(y^{0}\right)$.

Put $\hat{\eta}=\min \left\{\eta, \widehat{\vartheta}^{4}\right\}$ (here $\widehat{\vartheta}$ is the constant from Lemma 4.2). Note that the coefficients $\beta_{i}$ are bounded on the set $K_{\rho}\left(y^{0}\right) \cap\left\{y_{k}>\frac{\hat{\eta}}{3} \rho, k=1, \ldots, n\right\}$ and that, by (3.4), their $L_{n}$-norms over this set are bounded from above by some constant independent of $\rho$ and $y^{0}$. The "lemma on spreading of ink-stains" (see [3, Lemma 2.2]) allows us to obtain the estimate $v \geq \varkappa_{1} s$ on the set $K_{\left(1-\frac{\hat{\vartheta}^{3}}{2}\right) \rho}\left(y^{0}\right) \cap\left\{y_{k} \geq \frac{\hat{\vartheta}^{4}}{2} \rho, k=1, \ldots, n\right\}$ (here $\varkappa_{1}$ depends only on $n, \nu$, and $\left.\left(\lambda_{i}\right)\right)$.

Assume that the cube $K_{\left(1-\frac{\hat{\vartheta}^{3}}{2}\right) \rho}\left(y^{0}\right)$ intersects some coordinate hyperplane (let it be the hyperplane $\left.y_{1}=0\right)$. We consider all cubes $K_{\frac{\hat{\vartheta}^{2}}{2} \rho}$ that lie completely in the half-spaces $y_{k}>0, k=2, \ldots, n$, and lean out of the half-space $y_{1}>0$ at a depth of $\frac{\widehat{\vartheta}^{3}}{2} \rho$. Applying Lemma 4.2 to these cubes, we obtain the estimate $v \geq \frac{\varkappa_{1}}{2} s$ in the set $K_{\left(1-\frac{\hat{\vartheta}^{2}}{2}\right) \rho}\left(y^{0}\right) \cap\left\{\left|y_{1}\right| \leq \frac{\hat{\vartheta}^{4}}{2} \rho ; y_{k}>\frac{\hat{\vartheta}^{2}}{2} \rho, k=2, \ldots, n\right\}$. Then we again refer to the "lemma on spreading of ink-stains" (in the second orthant), obtaining the estimate $v \geq \varkappa_{2} s$ in the set $K_{\left(1-\frac{\hat{\vartheta}^{3}}{2}\right) \rho}\left(y^{0}\right) \cap\left\{y_{k} \geq \frac{\widehat{\vartheta}^{4}}{2} \rho, k=2, \ldots, n\right\}$ except for a "tunnel" of depth $\frac{\widehat{\vartheta}^{2}}{2} \rho$ in the neighborhood of the hyperplane $y_{1}=0$ (here $\varkappa_{2}$ also depends only on $n, \nu$, and $\left(\lambda_{i}\right)$ ).

Proceeding as above, after at most $n$ steps we obtain the estimate $v \geq 2 \varkappa s$ in the cube $K_{\left(1-\frac{\hat{\vartheta}^{3}}{2}\right) \rho}\left(y^{0}\right)$ except for a cross-shaped "tunnel" of depth $\widehat{\vartheta}^{2} \rho$ in the neighborhood of the hyperplanes $y_{k}=0, k=1, \ldots, n$. Finally, we apply Lemma 4.2 to cubes $K_{\frac{\rho}{2}}$ that lean out of the half-space $y_{k}>0$ at a depth of $\frac{\widehat{\vartheta}}{2} \rho$. This gives the estimate $v \geq \varkappa s$ in the cube $K_{\frac{\rho}{2}}\left(y^{0}\right)$.

To treat the general case, it suffices to note that at every step we reduce the estimate by a quantity of the form $c \rho^{\gamma}\left\|\frac{f_{+}}{\sqrt{\lambda}}\right\|_{n, K_{\rho}\left(y^{0}\right)}$, where a constant $c$ depends only on $n, \nu$, and $\left(\lambda_{i}\right)$. This gives (4.8).

Lemma 4.3 is a direct analog of [3, Lemma 2.3], and now the Hölder estimate for solutions of (3.3) can be deduced in a standard way; see [3, §2]. Returning to $x$-coordinates, we obtain an estimate for solutions of equation (1.1). We only formulate the final result.

Theorem 4.4. Suppose a function $u \in W_{n, \mathrm{loc}}^{2}(\Omega)$ satisfies equation (1.1), and $|u| \leq M_{0}$ in $\Omega$. Suppose that the coefficients in (1.1) satisfy conditions (1.2)-(1.4) and (3.1), and that $\frac{f}{\operatorname{det}(\Lambda)} \in L_{n}(\Omega)$. Then for any subdomain $\Omega^{\prime} \Subset \Omega$ we have

$$
\|u\|_{\mathcal{C}^{\hat{\gamma}}\left(\overline{\Omega^{\prime}}\right)} \leq M_{\widehat{\gamma}}
$$

where $\hat{\gamma}$ is completely determined by $n, \nu$, and the collection $\left(\lambda_{i}\right)$, while $M_{\hat{\gamma}}$ depends also on $M_{0},\left\|\frac{f}{\operatorname{det}(\Lambda)}\right\|_{n, \Omega}$, and $\operatorname{dist}\left(\Omega^{\prime}, \partial \Omega\right)$. 


\section{$\S 5$. Parabolic case}

In this section we consider equation (1.5) under the assumptions (1.2)-(1.4) and (3.1). In essence, the general line to establish the Hölder estimates is the same as in the preceding section. Therefore, here we only formulate the corresponding lemmas and give some hints to the proofs. We denote $\left.Q_{\rho}(x, t)=K_{\rho}(x) \times\right] t-\rho^{2}, t\left[, Q_{\rho}^{\prime}(\bar{x}, t)=K_{\rho}^{\prime}(\bar{x}) \times\right] t-\rho^{2}, t[$.

The proof of the next lemma repeats that of Lemma 2.3 word for word.

Lemma 5.1. Suppose $\varphi_{k} \in \mathcal{R}_{\alpha_{k}}, \alpha_{k}>-1, k=1, \ldots, n$. For measurable sets $E \subset \mathbb{R}^{n+1}$, define the measure

$$
|E|_{\star}=\int_{E} \varphi_{1}\left(\left|x_{1}\right|\right) \ldots \varphi_{n}\left(\left|x_{n}\right|\right) d x_{1} \ldots d x_{n} d t .
$$

Then for any $\varepsilon>0$ there exists $\delta>0$, depending only on $\varepsilon$ and the collection $\left(\varphi_{k}\right)$, such that for all cylinders $\left.Q_{\rho}\left(x^{0}, t^{0}\right) \subset K_{1} \times\right] 0, T[$ and an arbitrary measurable set $E$ we have

$$
\left|E \cap Q_{\rho}\left(x^{0}, t^{0}\right)\right| \leq \delta \cdot\left|Q_{\rho}\left(x^{0}, t^{0}\right)\right| \quad \Longrightarrow \quad\left|E \cap Q_{\rho}\left(x^{0}, t^{0}\right)\right|_{\star} \leq \varepsilon \cdot\left|Q_{\rho}\left(x^{0}, t^{0}\right)\right|_{\star} .
$$

After the coordinate transformation (3.2), equation (1.5) becomes

$$
\widetilde{\mathcal{M}} u \equiv \partial_{t} u-\sum_{i, j=1}^{n} \tilde{a}_{i j}(y, t) u_{y_{i} y_{j}}+\sum_{i=1}^{n} \tilde{a}_{i i}(y, t) \beta_{i}\left(y_{i}\right) u_{y_{i}}=f(y, t)
$$

(we keep the original notation for "transplanted" functions, as before).

In Lemmas 5.2-5.4 we suppose that the function $v \in W_{n+1}^{2,1}\left(K_{3 R_{0}} \times\right] 0, T[)$ after passage to the $y$-coordinates is nonnegative in the cylinder $Q_{\rho}\left(y^{0}, t^{0}\right) \subset(\widetilde{\Pi} \times] 0, T[)$ and satisfies the inequality $\widetilde{\mathcal{M}} v \geq-f(y, t)$ a.e.

Lemma 5.2. There exist constants $\tilde{\zeta} \in] 0,1[, \tilde{\eta} \in] 0,1\left[, \tilde{\gamma}>0\right.$, and $C_{6}>0$, depending only on $n, \nu$, and $\left(\lambda_{i}\right)$, such that if the inequality $\left|Q_{\rho}\left(y^{0}, t^{0}\right) \cap A_{s}^{v}\right| \geq(1-\widetilde{\zeta})\left|Q_{\rho}\left(y^{0}, t^{0}\right)\right|$ is fulfilled for some $s>0$, then

$$
v \geq \frac{s}{2}-C_{6} \rho^{\tilde{\gamma}}\left\|\frac{f_{+}}{\sqrt{\lambda}}\right\|_{n+1, Q_{\rho}\left(y^{0}, t^{0}\right)} \quad \text { in } \quad Q_{\tilde{\eta} \rho}\left(y^{0}, t^{0}\right) .
$$

Proof. The proof follows the lines of Lemma 4.1. Instead of the barrier function $W$ (see (4.2)), we introduce the function $\mathcal{W}(y, t)=W(y)+\frac{t^{0}-t}{\rho^{2}}$, and instead of the Aleksandrov maximum principle we use its parabolic version (see [17] and also [18, 19]). This gives an analog of inequality (4.3):

$$
v(y, t) \geq \operatorname{s\mathcal {W}}(y, t)-N_{7}\left|\Pi_{\rho}\left(x^{0}\right)\right|^{\frac{1}{n+1}} \cdot\left(s \frac{\left(\varepsilon\left|Q_{\rho}\left(y^{0}, t^{0}\right)\right|_{*}\right)^{\frac{1}{n+1}}}{\rho^{2}}+\left\|\frac{f_{+}}{\sqrt{\lambda}}\right\|_{n+1, Q_{\rho}\left(y^{0}, t^{0}\right)}\right),
$$

where $N_{7}$ depends only on $n, \nu$, and $\left(\lambda_{i}\right)$, and $\varepsilon=\frac{\left|Q_{\rho}\left(y^{0}, t^{0}\right) \backslash A_{s}^{v}\right|_{*}}{\left|Q_{\rho}\left(y^{0}, t^{0}\right)\right|_{*}}$. The rest of the proof runs practically without changes.

Lemma 5.3. There exist constants $\widetilde{\vartheta} \in] 0, \frac{1}{4}\left[\right.$ and $C_{7}>0$, depending only on $n, \nu$, and $\left(\lambda_{i}\right)$, such that if $0 \leq y_{m}^{0} \leq(1-\widetilde{\vartheta}) \rho$ for some $m$ (for definiteness, let $m=1$ ), and if for some $s>0$ the inequality $v \geq s$ is fulfilled on the set $\left\{\widetilde{\vartheta}^{2} \rho\right\} \times Q_{(1-\widetilde{\vartheta}) \rho}^{\prime}\left(\bar{y}^{0}, t^{0}\right)$, then

$$
\left.v \geq \frac{s}{2}-C_{7} \rho^{\tilde{\gamma}}\left\|\frac{f_{+}}{\sqrt{\lambda}}\right\|_{n+1, Q_{\rho}\left(y^{0}, t^{0}\right)} \quad \text { in the cylinder } \quad\right]-\widetilde{\vartheta}^{2} \rho, \widetilde{\vartheta}^{2} \rho\left[\times Q_{\widetilde{\vartheta}_{\rho}}^{\prime}\left(\overline{y^{0}}, t^{0}\right),\right.
$$

where $\widetilde{\gamma}$ is the constant from Lemma 5.2. 
Proof. The proof follows the lines of that of Lemma 4.2, but instead of the barrier function $\widehat{W}$ (see $(4.7)$ ) we introduce the function $\widehat{\mathcal{W}}(y, t)=\widehat{W}(y)+\frac{t^{0}-t}{\rho^{2}}$, and use the parabolic version of the Aleksandrov maximum principle.

Lemma 5.4. There exist constants $\tilde{\varkappa} \in] 0,1\left[\right.$ and $C_{8}>0$, depending only on $n, \nu$, and $\left(\lambda_{i}\right)$, such that if the inequality $\left|Q_{\frac{\rho}{2}}\left(y^{0}, t^{0}-\frac{3 \rho^{2}}{4}\right) \cap A_{s}^{v}\right| \geq(1-\widetilde{\zeta})\left|Q_{\frac{\rho}{2}}\left(y^{0}, t^{0}\right)\right|$ is fulfilled for some $s>0$, then

$$
v \geq \tilde{\varkappa} s-C_{8} \rho^{\tilde{\gamma}}\left\|\frac{f_{+}}{\sqrt{\lambda}}\right\|_{n+1, Q_{\rho}\left(y^{0}, t^{0}\right)} \quad \text { in } \quad Q_{\frac{\rho}{2}}\left(y^{0}, t^{0}\right)
$$

(here $\tilde{\zeta} \in] 0,1[$ and $\tilde{\gamma}>0$ are constants from Lemma 5.2 ).

Proof. The proof follows the lines of that of Lemma 4.3. Instead of [3, Lemma 2.2], at every step we should apply [3. Lemma 3.2], taking account of the fact that the coefficients $\beta_{i}$ are bounded on the set $Q_{\rho}\left(y^{0}, t^{0}\right) \cap\left\{y_{k}>\frac{\widetilde{\vartheta}^{4}}{3} \rho, k=1, \ldots, n\right\}$, and, by (3.4), their $L_{n+2}$-norms over this set are bounded from above by some constant independent of $\rho$ and $y^{0}$.

Lemma 5.4 is a direct analog of [3. Lemma 3.3], and now the Hölder estimates for solutions of equation (5.1) are deduced in a standard way; see [3, §3]. Returning to $x$-coordinates, we obtain an estimate for solutions of equation (1.5). We only formulate the final result.

Theorem 5.5. Suppose a function $u \in W_{n+1, l o c}^{2,1}(Q)$ satisfies equation (1.5), and $|u| \leq$ $M_{0}$ in $Q$. Suppose that the coefficients in (1.5) satisfy conditions (1.2)-(1.4) and (3.1), and that $\frac{f}{\operatorname{det}(\Lambda)} \in L_{n+1}(Q)$. Then for any subcylinder $\left.Q^{\prime}=\Omega^{\prime} \times\right] \delta, T\left[, \Omega^{\prime} \Subset \Omega\right.$, we have

$$
\|u\|_{\mathcal{C}_{\bar{\gamma}}\left(\overline{Q^{\prime}}\right)} \leq M_{\bar{\gamma}},
$$

where $\bar{\gamma}$ is completely determined by $n, \nu$, and the collection $\left(\lambda_{i}\right)$, while $M_{\bar{\gamma}}$, depends also on $M_{0},\left\|\frac{f}{\operatorname{det}(\Lambda)}\right\|_{n+1, Q}, \delta$, and $\operatorname{dist}\left(\Omega^{\prime}, \partial \Omega\right)$.

Remark 2. In (5.2), as usual, the Hölder norm is with respect to the parabolic metric

$$
d_{\operatorname{par}}\left(\left(x^{1}, t^{1}\right),\left(x^{2}, t^{2}\right)\right)=\left|x^{1}-x^{2}\right|+\left|t^{1}-t^{2}\right|^{1 / 2} .
$$

\section{§6. About approximative solutions}

If the coefficients in (1.1) are only measurable, in the general situation one cannot ensure the existence of a solution $u \in W_{n, \text { loc }}^{2}(\Omega)$ even in the uniformly elliptic case $\left(\lambda_{k} \equiv 1, k=1, \ldots, n\right)$. Therefore, approximative solutions of equation (1.1) are often considered. Namely, for $\left.\varepsilon \in] 0, \varepsilon_{0}\right]$ we set

$$
\lambda_{i}^{(\varepsilon)}(t)= \begin{cases}\lambda_{i}(t) & \text { if }|t| \geq \varepsilon \\ \lambda_{i}(\varepsilon) & \text { if }|t| \leq \varepsilon\end{cases}
$$

Next, we approximate the matrix $\widetilde{\mathcal{A}}$ in the sense of a.e. convergence with smooth matrix functions $\widetilde{\mathcal{A}}^{(\varepsilon)}$ that satisfy the uniform ellipticity condition with $\nu$ independent of $\varepsilon$. Finally, we introduce the matrices

$$
\begin{aligned}
\Lambda^{(\varepsilon)}(x) & =\operatorname{diag}\left\{\lambda_{1}^{(\varepsilon)}\left(\left|x_{1}\right|\right), \lambda_{2}^{(\varepsilon)}\left(\left|x_{2}\right|\right), \ldots, \lambda_{n}^{(\varepsilon)}\left(\left|x_{n}\right|\right)\right\}, \\
\mathcal{A}^{(\varepsilon)} & =\sqrt{\Lambda^{(\varepsilon)}} \tilde{\mathcal{A}}^{(\varepsilon)} \sqrt{\Lambda^{(\varepsilon)}},
\end{aligned}
$$


and consider the following sequence of uniformly elliptic equations with smooth coefficients:

$$
\mathcal{L}^{(\varepsilon)} u^{(\varepsilon)} \equiv-\sum_{i, j=1}^{n} a_{i j}^{(\varepsilon)}(x) u_{x_{i} x_{j}}^{(\varepsilon)}=f^{(\varepsilon)}(x)
$$

where $f^{(\varepsilon)}$ is a sequence of smooth functions such that $\left\|\frac{f^{(\varepsilon)}-f}{\operatorname{det}(\Lambda)}\right\|_{n, \Omega} \rightarrow 0$ as $\varepsilon \rightarrow 0$.

The standard elliptic theory (see, e.g., [20, Chapter III]) shows that equations (6.1) have classical solutions $u^{(\varepsilon)} \in \mathcal{C}^{\infty}(\Omega)$ for all $\varepsilon>0$. A function $u$ is called an approximative solution of equation (1.1) if there exists a subsequence of equations (6.1) and their solutions $u^{\left(\varepsilon_{k}\right)}$ convergent to $u$ a.e. in $\Omega$ as $\varepsilon_{k} \rightarrow 0$.

Remark 3. The result of 1 shows that the Dirichlet problem for a uniformly elliptic equation (1.1) has approximative solutions for any continuous boundary data. Moreover, solutions of the Dirichlet problems for equations (6.1) converge to the approximative solution uniformly in $\bar{\Omega}$. However, in [21, it was proved (see also [22]) that if $n \geq 3$, then, even for a uniformly elliptic equation (1.1), an approximative solution of the Dirichlet problem can depend on the sequence $\widetilde{\mathcal{A}}^{(\varepsilon)}$.

In this section we establish the Hölder property for an arbitrary approximative solution of equation (1.1) under the assumptions (1.2)-(1.4), (3.1). To proceed, we introduce a coordinate transformation by formula (3.2) with $\lambda_{k}^{(\varepsilon)}$ in place of $\lambda_{k}$ and denote by $\widetilde{\Pi}^{(\varepsilon)}$ the cuboid that is the image of $K_{3 R_{0}}$ under this transformation. Note that in the new coordinate system the operator $\mathcal{L}^{(\varepsilon)}$ reads as follows:

$$
\widetilde{\mathcal{L}}^{(\varepsilon)} u \equiv-\sum_{i, j=1}^{n} \widetilde{a}_{i j}^{(\varepsilon)}(y) u_{y_{i} y_{j}}+\sum_{i=1}^{n} \widetilde{a}_{i i}^{(\varepsilon)}(y) \beta_{i}^{(\varepsilon)}\left(y_{i}\right) u_{y_{i}},
$$

where $\beta_{k}^{(\varepsilon)}\left(y_{k}\right)=\frac{\lambda_{k}^{\prime}\left(x_{k}\right)}{2 \sqrt{\lambda_{k}\left(x_{k}\right)}} \cdot \chi_{[\varepsilon,+\infty[}\left(\left|x_{k}\right|\right)$.

Lemma 6.1. Let $\rho>0$. There exists $\widehat{\varepsilon}>0$, depending only on $\rho, n, \nu$, and the collection $\left(\lambda_{i}\right)$, such that for $0<\varepsilon \leq \hat{\varepsilon}$ the following is true.

Suppose that a function $v \in \mathcal{C}^{2}\left(\widetilde{\Pi}^{(\varepsilon)}\right)$ is nonnegative in the cube $K_{\rho}\left(y^{0}\right) \subset \widetilde{\Pi}^{(\varepsilon)}$ and satisfies the inequality $\widetilde{\mathcal{L}}^{(\varepsilon)} v \geq-f^{(\varepsilon)}(y)$. If the inequality $\left|K_{\frac{\rho}{2}}\left(y^{0}\right) \cap A_{s}^{v}\right| \geq\left(1-\frac{\zeta}{2}\right)\left|K_{\frac{\rho}{2}}\left(y^{0}\right)\right|$ is fulfilled for some $s>0$, then

$$
v \geq \frac{\varkappa}{2} s-2 C_{5} \rho^{\gamma}\left\|\frac{f_{+}^{(\varepsilon)}}{\sqrt{\lambda^{(\varepsilon)}}}\right\|_{n, K_{\rho}\left(y^{0}\right)} \quad \text { in } \quad K_{\frac{\rho}{2}}\left(y^{0}\right)
$$

(here $\zeta$ and $\gamma$ are constants from Lemma 4.1, and $\varkappa, C_{5}$ are constants from Lemma 4.3).

Proof. The proof repeats the lines of $\S 4$. It suffices to note that all the quantities in the proofs of Lemmas 4.1-4.3 tend to their limits uniformly as $\varepsilon \rightarrow 0$.

Lemma 6.1 implies that if a nonnegative function $v$ in the cube $K_{\rho}\left(y^{0}\right) \subset \widetilde{\Pi}$ satisfies the inequality $\widetilde{\mathcal{L}} v \geq-f(y)$ in the approximative sense, then the statement of Lemma 4.3 holds true for that function (possibly, after changing on a zero measure set), with the replacement $\zeta \rightarrow \frac{\zeta}{2}, \varkappa \rightarrow \frac{\varkappa}{2}, C_{5} \rightarrow 2 C_{5}$. Since all further arguments from [3, §2] do not require the smoothness of functions, Theorem 4.4 remains valid for approximative solutions. 
Approximative solutions of equation (1.5) can be defined in a similar way. Theorem 5.5 remains valid for them.

I am thankful to Yu. A. Alkhutov who kindly provided me with the manuscript of the paper [12].

\section{REFERENCES}

[1] N. V. Krylov and M. V. Safonov, A property of the solutions of parabolic equations with measurable coefficients, Izv. Akad. Nauk SSSR Ser. Mat. 44 (1980), no. 1, 161-175; English transl. in Math. USSR-Izv. 16 (1981), no. 1. MR0563790 (83c:35059)

[2] M. V. Safonov, Harnack's inequality for elliptic equations and Hölder property of their solutions, Zap. Nauchn. Sem. Leningrad. Otdel. Mat. Inst. Steklov. (LOMI) 96 (1980), 272-287; English transl. in J. Soviet math. 21 (1983), no. 5. MR0579490 (82b:35045)

[3] O. A. Ladyzhenskaya and N. N. Ural'tseva, Estimates of the Hölder constant for functions satisfying a uniformly elliptic or uniformly parabolic quasilinear inequality with unbounded coefficients, Zap. Nauchn. Sem. Leningrad. Otdel. Mat. Inst. Steklov. (LOMI) 147 (1985), 72-94; English transl., J. Soviet Math. 37 (1987), no. 1, 837-851. MR0821476 (87e:35090)

[4] E. B. Fabes and D. W. Stroock, The $L_{p}$-integrability of Green's functions and fundamental solutions for elliptic and parabolic equations, Duke Math. J. 51 (1984), no. 4, 997-1016. MR0771392 (86g:35057)

[5] N. V. Krylov, Boundary inhomogeneous elliptic and parabolic equations in a domain, Izv. Akad. Nauk SSSR Ser. Mat. 47 (1983), no. 1, 75-108; English transl., Math. USSR-Izv. 22 (1984), no. 1, 67-98. MR0688919 (85g:35046)

[6] J. J. Kohn and L. Nirenberg, Degenerate elliptic-parabolic equations of second order, Comm. Pure Appl. Math. 20 (1967), 797-872. MR0234118 (38:2437)

[7] P. Daskalopoulos and K. Lee, Free-boundary regularity on the focusing problem for the Gauss curvature flow with flat sides, Math. Z. 237 (2001), no. 4, 847-874. MR.1854093 (2002g:53114)

[8] R. Hamilton, Worn stones with flat sides, A Tribute to Ilya Bakelman (College Station, TX, 1993), Discourses Math. Appl., vol. 3, Texas A \& M Univ., College Station, TX, 1994, pp. 69-78. MR:1423370 (97m:52008)

[9] P. Daskalopoulos and R. Hamilton, The free boundary in the Gauss curvature flow with flat sides, J. Reine Angew. Math. 510 (1999), 187-227. MR.1696096 (2000g:53081)

[10] P. Daskalopoulos and K. Lee, Worn stones with flat sides all time regularity of the interface, Invent. Math. 156 (2004), 445-493. MR2061326 (2005c:53084)

[11] _ Hölder regularity of solutions of degenerate elliptic and parabolic equations, J. Funct. Anal. 201 (2003), no. 2, 341-379. MR1986693 (2004h:35024)

[12] Yu. A. Alkhutov, On the Hölder continuity of solutions of second-order degenerate elliptic equations in nondivergence form, Dokl. Akad. Nauk 413 (2007), no. 3, 295-300. (Russian) MR2456743 (2009g:35094)

[13] , Harnack's inequality for solutions of a class of degenerate elliptic equations, Internat. Conf. on Differential Equations and Dynamical Systems (Suzdal', 2006): Thesis, pp. 22-24. (Russian)

[14] E. Seneta, Regularly varying functions, Lecture Notes in Math., vol. 508, Springer-Verlag, BerlinNew York, 1976. MR0453936 (56:12189)

[15] E. Lieb and M. Loss, Analysis, Grad. Stud. Math., vol. 14, Amer. Math. Soc., Providence, RI, 1997. MR 1415616 (98b:00004)

[16] A. D. Aleksandrov, Uniqueness conditions and bounds for the solution of the Dirichlet problem, Vestnik Leningrad. Univ. Ser. Mat. Mekh. Astronom. 1963, vyp. 3, 5-29. (Russian) MR0164135 (29:1434)

[17] N. V. Krylov, Sequences of convex functions, and estimates of the maximum of the solution of a parabolic equation, Sibirsk. Mat. Zh. 17 (1976), no. 2, 290-303; English transl., Siberian Math. J. 17 (1976), no. 2, 226-236. MR0420016 (54:8033)

[18] A. I. Nazarov and N. N. Ural'tseva, Convex-monotone hulls and an estimate of the msximum of the solution of a parabolic equation, Zap. Nauchn. Sem. Leningrad. Otdel. Mat. Inst. Steklov. (LOMI) 147 (1985), 95-109; English transl., J. Soviet Math. 37 (1987), no. 1, 851-859. MR0821477 (87h:35039)

[19] K. Tso, On an Aleksandrov-Bakel'man type maximum principle for second-order parabolic equations, Comm. Partial Differential Equations 10 (1985), no. 5, 543-553. MR0790223 (87f:35031)

[20] O. A. Ladyzhenskaya and N. N. Ural'tseva, Linear and quasilinear equations of elliptic type, 2nd ed., Nauka, Moscow, 1973; English transl. of 1st ed., Acad. Press, New York-London, 1968. MR0509265 (58:23009) MR0244627 (39:5941) 
[21] N. S. Nadirashvili, Nonuniqueness in the martingale problem and the Dirichlet problem for uniformly elliptic equations, Ann. Scuola Norm. Sup. Pisa Cl. Sci. (4) 24 (1997), no. 3, 537-549. MR 1612401 (99b:35042)

[22] M. V. Safonov, Nonuniqueness for second-order elliptic equations with measurable coefficients, SIAM J. Math. Anal. 30 (1999), no. 4, 879-895. MR1684729(2000c:35035)

Department of Mathematics and Mechanics, St. Petersburg State University, UniverSitetskiI Prospekt 28, Petrodvorets, St. Petersburg 198504, Russia

E-mail address: al.il.nazarov@gmail.com

Received 8/SEP/2008

Translated by THE AUTHOR 